\title{
Implications of losing Hermiticity in quantum mechanics
}

\author{
N. Bebiano* and J. da Providência ${ }^{\dagger}$ \\ January 17, 2017
}

\begin{abstract}
In this note, we revisit a system of one self-interacting boson, described by a non-Hermitian Hamiltonian $H$ acting on an infinite dimensional Hilbert space $\mathcal{H}$. We determine the eigenfunctions of the Hamiltonian and of its adjoint, which constitute complete biorthogonal sets. The probabilistic interpretation of quantum mechanics is not compatible with the metric inherited from $\mathcal{H}$, and attempts to overcome this problem are presented. Consequences of losing self-adjointness in the quantum mechanical context are discussed and the necessity of a careful mathematical analysis of unbounded operators is emphasized.
\end{abstract}

"In spite of the quasi-Hermiticity (without bounded inverse of $Q$ ), there is for instance no hope of building functional calculus that would follow more or less the same pattern of the self-adjoint operators."

AMS subject classification: $34 \mathrm{~K} 08,47-02,46 \mathrm{~N} 50$

Dieudonné

Key words: Hamiltonian, unbounded operator, Complete biorthogonal sets, Riesz basis, Harmonic oscillator

\section{Introduction}

In quantum mechanics, the states of a particle, or system of particles, are represented by vectors in a separable Hilbert space $\mathcal{H}$, endowed with an inner product $\langle\cdot, \cdot\rangle$ and related norm $\|\cdot\|$. Measurable physical quantities are represented by self-adjoint, or Hermitian (in the terminology of von Neumann), operators in

${ }^{*}$ CMUC, University of Coimbra, Department of Mathematics, P 3001-454 Coimbra, Portugal (bebiano@mat.uc.pt)

${ }^{\dagger}$ CFisUC, Department of Physics, University of Coimbra, P 3004-516 Coimbra, Portugal (providencia@teor.fis.uc.pt) 
$\mathcal{H}$. Such operators are called observables. This mathematical structure is the scenario we will be concerned with.

The states of the quantum system are represented by vectors in $\mathcal{H}$, which evolve in time according to the time dependent Schrödinger equation

$$
i \frac{\mathrm{d}}{\mathrm{d} t} \psi=H \psi
$$

where $\psi=\psi(t)$ is the time dependent wave vector. In this context, the eigenvalues $E_{n}$ of $H$ must be real and its eigenvectors $\psi_{n}$ must constitute a complete orthogonal system, and they can be easily orthonormalized. The time dependent Schrödinger equation is solved by expanding the initial state vector as

$$
\psi(0)=\sum_{n=0}^{\infty} c_{n} \psi_{n}, \quad c_{n} \in \mathbb{C}
$$

and, at the instant $t$, the solution of the equation is given by

$$
\psi(t)=\sum_{n=0}^{\infty} c_{n} \mathrm{e}^{-i E_{n} t} \psi_{n} .
$$

The relevance in physics of non-Hermitian operators with real eigenvalues is being widely recognized not only in quantum mechanics, but also in other areas of quantum physics such as quantum fluid dynamics, quantum optics, quantum field theory, and so on [5]. Initially, the literature on these topics was mainly written by physicists for physicists, but certain mathematical contents deserved the mathematicians attention $[5,13]$, and now this is a crossed specialization area of research of both scientific communities.

We will be mainly concerned with the following questions.

Firstly, in finite dimensions, self-adjointness of the Hamiltonian ensures the existence of real eigenvalues and orthogonal eigenvectors, a fundamental issue for the probabilistic interpretation of quantum mechanics. Eigensystems of nonself-adjoint operators can show very wild properties and they present a very different behaviour from the self-adjoint setup [13]. The occurrence, in the description of certain quantum systems, of non-self-adjoint Hamiltonians possessing real eigenvalues, motivates the study of the implications of non-self-adjointness in the mathematical apparatus of quantum mechanics.

Secondly, a Hamiltonian operator, whether it is self-adjoint or not, is in general unbounded. As a consequence, domain problems, among others, arise out of this feature. The unbounded nature of several operators appearing in this context requires some attack subtleties. While quantum theory is well understood and developed for systems involving bounded metric operators with a bounded inverse, the emergence of unbounded metric operators causes difficulties and places challenging problems. 
Unbounded operators exhibit pathological properties, such as they can map an orthonormal basis into a set that may not form any kind of reasonable basis (see $[4,6,7,8,9,11,10,13,16,17]$ and references therein).

One of our main purposes is to study some implications of losing self-adjointness. Concretely, our goal is to investigate spectral properties of a quantum system described by a non-Hermitian Hamiltonian, acting on an infinite dimensional separable Hilbert space. The system is constituted by a self-interacting boson and the Hamiltonian $H$ has a real and discrete spectrum. The existence of a basis of eigenfunctions, crucial for the interpretation of quantum mechanics, is discussed. One second goal is to expose some distinctive features of unbounded operators. It should be stressed that the problems identified in our simple model appear in several situations, so it represents a good illustration of the problematics in the area.

\section{The non-Hermitian shifted harmonic oscilla- tor}

Let us consider the Hilbert space $\mathcal{H}=L^{2}(\mathbb{R})$ of square integrable functions in one real variable, endowed with the standard inner product

$$
\langle f, g\rangle=\int_{-\infty}^{+\infty} g(x) \overline{f(x)} \mathrm{d} x .
$$

Let $\mathcal{D}(H)$ denote an unspecified domain of an operator $H$. Consider the standard unbounded bosonic operators $a: \mathcal{D}(a) \rightarrow \mathcal{D}(a)$ and $a^{*}: \mathcal{D}\left(a^{*}\right) \rightarrow \mathcal{D}\left(a^{*}\right)$, expressed as

$$
\begin{aligned}
& a=-\frac{i}{\sqrt{2}} \frac{\mathrm{d}}{\mathrm{d} x}-\frac{i}{\sqrt{2}} x=\frac{1}{\sqrt{2}} p-\frac{i}{\sqrt{2}} x, \\
& a^{*}=-\frac{i}{\sqrt{2}} \frac{\mathrm{d}}{\mathrm{d} x}+\frac{i}{\sqrt{2}} x=\frac{1}{\sqrt{2}} p+\frac{i}{\sqrt{2}} x
\end{aligned}
$$

where the Hermitian operator $p$ is the so called momentum operator

$$
-i \frac{\mathrm{d}}{\mathrm{d} x}: \mathcal{D}(p) \rightarrow \mathcal{D}(p)
$$

and $x: \mathcal{D}(x) \rightarrow \mathcal{D}(x)$ is the position operator $x$.

We introduce the well-known non-Hermitian harmonic oscillator [5, pp. 145152] described by the Hamiltonian $H: \mathcal{D}(H) \rightarrow \mathcal{D}(H)$,

$$
H=\frac{1}{2}\left(p^{2}+x^{2}\right)+i \alpha \sqrt{2} x-\frac{1}{2}, \quad \alpha \in \mathbb{R} .
$$


This non-Hermitian operator is invariant under a simultaneous space reflection $\mathcal{P}(x \rightarrow-x)$ and time reversal $\mathcal{T}$ (complex conjugation). Moreover, it is unbounded and pertinent problems arise with unbounded operators. $\mathcal{P} \mathcal{T}$-symmetric models have applications in several domains of physics; namely in optics, electromagnetism, super conductivity, solid state, Bose-Einstein condensates, etc. For $\mathcal{P} \mathcal{T}$-symmetric Hamiltonians, see ref. [9] and references therein.

\subsection{Some words on $\mathcal{P} \mathcal{T}$-symmetry}

In the turning of the last century, $\mathcal{P} \mathcal{T}$-symmetry became a challenging issue (see $[9,14,16,18]$ and their references). It is consensual that a quantum mechanical interpretation of $\mathcal{P} \mathcal{T}$-symmetry must be implemented through an invertible similarity transformation $R$,

$$
h:=R_{H} R^{-1},
$$

where $h$ is a self-adjoint operator, $h=h^{*}$.

This relation is closely related to the so called quasi-Hermiticity

$$
Q H=H^{*} Q
$$

where $Q$ is a positive operator called a metric operator [15]. A Hamiltonian $H$ with this property is called quasi-Hermitian, because it is Hermitian with respect to the inner product induced by the metric operator $Q$,

$$
\langle\cdot, Q \cdot\rangle=\left\langle Q^{1 / 2} \cdot Q^{1 / 2} \cdot\right\rangle .
$$

It is not easy to give a good meaning of (2) when considering the respective domains. The operator equality (2) requires that the operator domains $\mathcal{D}(Q H)$ and $\mathcal{D}\left(H^{*} Q\right)$ are equal and in addition the identity

$$
Q H \psi=H^{*} Q \psi
$$

holds for every $\psi \in \mathcal{D}(Q H) \cap \mathcal{D}\left(H^{*} Q\right)$. It should be stressed that if $Q$ is invertible and bounded, then fundamental properties of self-adjoint operators are valid for $H$, e.g., real spectrum, spectral stability with respect to perturbations, etc. Otherwise, if the metric is singular (i.e. noninvertible, unbounded, or unboundedly invertible), the mentioned properties have no guaranteed validity, and this may cause serious problems in the behavior of $H$.

\subsection{Domain considerations}

Let us initially chose the domain

$$
\mathcal{D}=\left\{f(x) \mathrm{e}^{-x^{2} / 2}: f(x) \text { is a polynomial in } x\right\},
$$


which is dense in $L^{2}(\mathbb{R})$.

It is immediate to conclude that the operator $p:=-i d / d x$, with a domain consisting of "nice" functions (like for instance the set $\mathcal{D}$ ), is symmetric. This symmetry implies that

$$
\mathcal{D}(p) \subset \mathcal{D}\left(p^{*}\right),
$$

But there is a big difference between symmetric and self-adjoint operators, the latter meaning that actually $p=p^{*}$ and $\mathcal{D}(p)=\mathcal{D}\left(p^{*}\right)$, that is, an operator is self-adjoint if it is symmetric and its domain coincides with the domain of its adjoint. There is even a big difference between self-adjoint and closed symmetric operators ([5], pp.60-88). Of course, such subtleties do not arise for bounded operators, but they are crucial in the setup of unbounded operators.

In our case, $p:=-i d / d x$ is self-adjoint if its domain is correctly chosen in the context of the Sobolev space $H^{1}$, but this is definitely not the case, if we consider our much smaller set $\mathcal{D}$. The operator is not even closed on this set.

In applied research, the specification of operator domains is often missing. We must choose the most convenient closed domain among all possible ones. In the majority of "applied situations", that domain must be different from the operator maximal domain. In what concerns our model, we recall that weighted shifts are not closed, this is a basic fact, nevertheless they are closable.

It is well-known that $H$ is a closed operator on its maximal domain

$$
\operatorname{dom}(H)=\left\{\psi \in L^{2}(\mathbb{R}):-\psi^{\prime \prime}+x^{2} \psi+i x \psi \in L^{2}(\mathbb{R})\right\} .
$$

It may be easily shown that $H$ is a shifted non-Hermitian operator. In fact, the bosonic operators $a, a^{*}$ satisfy the following commutation relation

$$
\left[a, a^{*}\right]=a a^{*}-a^{*} a=\mathbf{1},
$$

where $\mathbf{1}$ is the identity operator in $\mathcal{H}$ (this means that $\left[a, a^{*}\right] f=f$ for any $f \in \mathcal{D}$ ). In terms of $a^{*}, a$, the non-Hermitian operator $H$, is expressed as

$$
H=a^{*} a+\alpha\left(a^{*}-a\right), \quad \alpha \in \mathbb{R},
$$

that is, a shifted non-Hermitian operator. Its Cartesian decomposition is $H=$ $\Re A+i \Im A$, where $\Re H=a^{*} a$ and $\Im H=-i \alpha\left(a-a^{*}\right)$. Obviously, $\Re H$ is positive definite. As a consequence, the numerical range of $H$, denoted and defined as

$$
W(H):=\{\langle H \psi, \psi\rangle: \psi \in \mathcal{D}(H),\|\psi\|=1\},
$$

is a subset of a sector in the closed right-half plane, which gives an accessible spectral region for $H$.

Next, we determine an orthonormal basis for $\mathcal{H}$. Assume that the nonzero vector $\Phi_{0} \in \mathcal{D}$ is the vacuum of $a$,

$$
a \Phi_{0}=0 .
$$


We easily find that $\Phi_{0}$ is the Gaussian

$$
\Phi_{0}(x)=\frac{1}{\pi^{1 / 4}} \mathrm{e}^{-x^{2} / 2} \in \mathcal{D} .
$$

Then, the eigenvectors $\Phi_{n}$ of the number operator $N_{0}=a^{*} a$, are

$$
\Phi_{n}=a^{* n} \Phi_{0}=f_{n}(x) \mathrm{e}^{-x^{2} / 2} \in \mathcal{D}, \quad n=0,1,2, \ldots,
$$

with $f_{n}(x)$ proportional to the $n t h$ Hermite polynomial in $x$.

These eigenvectors constitute an orthogonal basis of $\mathcal{H}$, orthogonality being easily shown, as

$$
\left\langle\Phi_{n}, \Phi_{m}\right\rangle=\delta_{m, n} n !\left\langle\Phi_{0}, \Phi_{0}\right\rangle=\delta_{m, n} n !,
$$

where $\delta_{m n}$ is the Kroenecker symbol $\left(\delta_{m n}=1\right.$ if $m=n$ and 0 otherwise). The basis may obviously be orthonormalized in a standard way:

$$
e_{0}(x)=\frac{1}{\pi^{1 / 4}} \mathrm{e}^{-x^{2} / 2}, \quad e_{n}(x)=\frac{1}{\sqrt{2^{n} n ! \pi^{1 / 2}}} H_{n}(x) \mathrm{e}^{-x^{2} / 2}, \quad n=1,2, \ldots
$$

where $H_{n}(x)$ is the $n t h$ Hermite polynomial in $x$.

\subsection{Eigenvectors of the Hamiltonian and of its adjoint}

We have that

$$
\left[H, a^{*}\right]=a^{*}-\alpha, \quad[H, a]=-a-\alpha, \quad[H, \mathbf{1}]=0 .
$$

In order to determine the eigenvalues and eigenvectors of $H$, we consider a (real) linear combination of $a^{*}, a, \mathbf{1}$, the generators of an Heisenberg algebra,

$$
\theta=x a^{*}+y a+z \mathbf{1}
$$

The requirement

$$
[H, \theta]=x a^{*}-y a-\alpha(x+y)=\lambda\left(x a^{*}+y a+z \mathbf{1}\right), \quad \lambda \in \mathbb{R},
$$

leads to the following real linear system

$$
\begin{aligned}
& x=\lambda x \\
& -y=\lambda y \\
& -\alpha(x+y)=\lambda z .
\end{aligned}
$$

Equivalently, in matrix form, we get

$$
\left[\begin{array}{ccc}
1 & 0 & 0 \\
0 & -1 & 0 \\
-\alpha & -\alpha & 0
\end{array}\right]\left[\begin{array}{l}
x \\
y \\
z
\end{array}\right]=\lambda\left[\begin{array}{l}
x \\
y \\
z
\end{array}\right] .
$$


The eigenvalues of the above linear system are readily obtained,

$$
\lambda=1,-1,0,
$$

and the associated eigenvectors are $(1,0,-1)^{T},(0,1,1)^{T},(0,0,1)^{T}$, respectively.

These eigenvectors allow the introduction of the operators $\Theta^{\ddagger}: \mathcal{D}\left(\Theta^{\ddagger}\right) \rightarrow$ $\mathcal{D}\left(\Theta^{\ddagger}\right)$ and $\Theta: \mathcal{D}(\Theta) \rightarrow \mathcal{D}(\Theta)$, defined, respectively, by

$$
\Theta^{\ddagger}=a^{*}-\alpha, \quad \Theta=a+\alpha,
$$

and satisfying the canonical commutation rule

$$
\left[\Theta, \Theta^{\ddagger}\right]=1 \text {. }
$$

The vacuum of $\Theta$ is given by the nonzero vector $\Psi_{0} \in \mathcal{D}$ such that

$$
\Theta \Psi_{0}=0,
$$

that is, $\Psi_{0}=\exp \left(-(x+i \sqrt{2} \alpha)^{2} / 2\right)$. Then, the eigenvectors of $H$ are

$$
\Psi_{n}=\Theta^{\ddagger n} \Psi_{0}=H_{n}(x+i \sqrt{2} \alpha) \mathrm{e}^{-(x+i \sqrt{2} \alpha)^{2} / 2},
$$

where $H_{n}(z)$ are polynomials in $z$ of degree $n$, with real coefficients, obtained from the polynomials in (4) by changing $x$ into $x+i \sqrt{2} \alpha$.

The eigenvectors of the adjoint $H^{*}: \mathcal{D}\left(H^{*}\right) \rightarrow \mathcal{D}\left(H^{*}\right)$, denoted by $\tilde{\Psi}_{n}$, can be similarly determined. Let the nonzero vector $\tilde{\Psi}_{0} \in \mathcal{D}$ denote the vacuum of $\Theta^{\ddagger *}$,

$$
\Theta^{\ddagger *} \tilde{\Psi}_{0}=0 .
$$

By (1), we find

$$
\left(\frac{\mathrm{d}}{\mathrm{d} x}+x-i \sqrt{2} \alpha\right) \tilde{\Psi}_{0}=0
$$

and so

$$
\tilde{\Psi}_{0}(x)=\exp \left(-(x-i \sqrt{2} \alpha)^{2} / 2\right), \quad n=0,1,2, \ldots
$$

Then we easily obtain the eigenvectors of $H^{*}$ :

$$
\tilde{\Psi}_{n}=\Theta^{* n} \tilde{\Psi}_{0}=H_{n}(x-i \sqrt{2} \alpha) \mathrm{e}^{-(x-i \sqrt{2} \alpha)^{2} / 2},
$$

where $H_{n}(z)$ are polynomials of degree $n$ in $z$, with real coefficients. The eigenvectors of $H^{*}$ and $H$ constitute biorthogonal systems of vectors, as

$$
\left\langle\tilde{\Psi}_{n}, \Psi_{m}\right\rangle=\delta_{m, n} n !\left\langle\tilde{\Psi}_{0}, \Psi_{0}\right\rangle, \quad m, n=0,1,2, \ldots
$$

In fact

$$
\begin{aligned}
& \left\langle\tilde{\Psi}_{n}, \Psi_{m}\right\rangle=\int_{-\infty}^{+\infty} \Psi_{m}(x) \overline{\tilde{\Psi}_{n}(x)} \mathrm{d} x=\int_{-\infty}^{+\infty} \Phi_{m}(x+i \sqrt{2} \alpha) \overline{\Phi_{n}(x-i \sqrt{2} \alpha)} \mathrm{d} x \\
& =\int_{-\infty}^{+\infty} \Phi_{m}(x+i \sqrt{2} \alpha) \Phi_{n}(x+i \sqrt{2} \alpha) \mathrm{d} x=n ! \delta_{m n} .
\end{aligned}
$$

Under a suitable normalization, we obtain two biorthonormal systems of vectors. 


\subsection{Eigenvalues of $H$ and $H^{*}$}

Next, we determine the eigenvalues of $H$ and $H^{*}$. From (5) it follows that we have

$$
H=\Theta^{\ddagger} \Theta+\alpha^{2} \mathbf{1},
$$

implying that the eigenvalues of $H$ are given by

$$
n+\alpha^{2}, \quad n=0,1,2, \ldots,
$$

the eigenvalue of $H$ associated with the eigenvector $\Psi_{n}$ being $n+\alpha^{2}$, as a consequence of (6),

$$
H \Psi_{n}=\left(n+\alpha^{2}\right) \Psi_{n} .
$$

Thus, all the eigenvalues of $H$ are real and simple. From (7) it follows that

$$
H^{*}=\Theta^{*} \Theta^{\ddagger *}+\alpha^{2} \mathbf{1},
$$

implying that eigenvalues of $H^{*}$ are given by (8), the eigenvalue $n+\alpha^{2}$ of $H^{*}$ being associated with the eigenvector $\tilde{\Psi}_{n}$

$$
H^{*} \tilde{\Psi}_{n}=\left(n+\alpha^{2}\right) \tilde{\Psi}_{n} .
$$

The operator $\Theta^{\ddagger}$ increases the eigenvalues of $H$ by one unit, because

$$
\Theta^{\ddagger} \Psi_{n}=\Psi_{n+1},
$$

so $\Theta^{\ddagger}$ is said to be a creation operator. On the other hand, the operator $\Theta$ decreases the eigenvalues of $H$ by one unit, because, keeping in mind that $[H, \Theta]=$ $-\Theta$, we have that

$$
\begin{aligned}
& \Theta \Psi_{n}=\Theta \Theta^{\ddagger n} \Psi_{0}=\left[\Theta, \Theta^{\ddagger n}\right] \Psi_{0}+\Theta^{\ddagger n} \Theta \Psi_{0}=n \Theta^{\ddagger(n-1)} \Psi_{0}+0 \\
& =n \Psi_{n-1}, \quad n>0,
\end{aligned}
$$

so $\Theta$ is said to be an annihilation operator.

From $\left[H, \Theta^{\ddagger}\right]=1$ and $[H, \Theta]=-1$, we conclude that $\left[H^{*}, \Theta^{\ddagger *}\right]=-1$ and $\left[H^{*}, \Theta^{*}\right]=1$. Thus, the creation and annihilation operators for $H^{*}$ are, respectively,

$$
\Theta^{*} \text { and } \Theta^{\ddagger *} \text {, }
$$

because, when acting on an eigenvector of $H^{*}$, the operator $\Theta^{*}$ increases the respective eigenvalue by 1 , while $\Theta^{\ddagger *}$ decreases the respective eigenvalue by the same amount. We recall that creation and annihilation operators are unbounded operators.

The operators $\Theta^{\ddagger}, \Theta$ do not describe bosons, as $\left[\Theta, \Theta^{\ddagger}\right]=1$ with $\Theta^{*} \neq \Theta^{\ddagger}$, but they describe $\mathcal{D}$-pseudo bosons (cf. [1]) because

$$
\left(\Theta \Theta^{\ddagger}-\Theta^{\ddagger} \Theta\right) f=f \quad \text { for all } f \in \mathcal{D} .
$$

The respective pseudo-bosonic number operator is defied by $N=\Theta^{\ddagger} \Theta$. 


\subsection{Eigenbases}

It may be shown that the set $\left\{\Psi_{n}\right\}$ of the eigenvectors of the non-Hermitian Hamiltonian is complete, that is, 0 is the only vector orthogonal to all its vectors. An analogous situation holds for $\left\{\tilde{\Psi}_{n}\right\}$, the eigenvectors of $H^{*}$.

In the present case, the sets $\left\{\Psi_{n}\right\}$ and $\left\{\widetilde{\Psi}_{n}\right\}$ do not constitute bases for $\mathcal{H}$ [1], essentially because the eigenvectors $\Psi_{n}$ and $\tilde{\Psi}_{n}$ are given by polynomials multiplying a shifted gaussian, and so

$$
\lim _{n}\left\|\Psi_{n}\right\|=\lim _{n}\left\|\tilde{\Psi}_{n}\right\|=\infty
$$

For more details, see [1].

When $\mathcal{H}$ is infinite dimensional, completeness of $\left\{\Psi_{n}\right\}$ is equivalent to $\left\{\Psi_{n}\right\}$ being a basis if $\left\{\Psi_{n}\right\}$ is an orthonormal set. Nevertheless, in general, when orthonormality does not hold, completeness by its own does not ensure basicity.

We recall that completeness of a non-orthogonal family $\left\{\Psi_{n}\right\}$ does not imply that any $\psi \in \mathcal{H}$ has a unique expansion of the form $\psi=\sum_{n} c_{n} \Psi_{n}$. The existence of an eigenbasis for $H$ (or $H^{*}$ ) would be important from the physical point of view, because it would mean that that any vector in $\mathcal{H}$ would represent a physical state, and so it could be uniquely written as a linear combination of the eigenvectors, as required by the Superposition Principle.

A simple reminder: $\left\{\Psi_{n}\right\}$ is a (Schauder or conditional) basis of $\mathcal{H}$ if any $\psi \in \mathcal{H}$, may be written as

$$
\sum_{n=0}^{\infty} c_{n} \Psi_{n}
$$

for a unique set of scalars $c_{n}$, where the infinite sum is understood as a limit in the strong topology of $\mathcal{H}$.

It is known that eingenfunctions of a Hamiltonian $H$ with discrete spectrum forms a Riesz basis if and only if $H$ is quasi-Hermitian with bounded and boundedly invertible metric [13, Proposition 4]. If $\left\{\Psi_{n}\right\}$ is a Riesz (or unconditional) basis of $\mathcal{H}$, a unique representation of the form (10) is ensured, and this fact is crucial in the quantum mechanical perspective. It should be noticed that, like the spectrum, Riesz basicity property is not preserved by unbounded transformations.

Neither $\left\{\Psi_{n}\right\}$ nor $\left\{\tilde{\Psi}_{n}\right\}$ are Riesz bases. It should be stressed that this is not a consequence of the chosen normalization, but an inevitable consequence of $H$ being non self-adjoint and possessing a nontrivial pseudospectrum (see next section). Nevertheless, this does not exclude the possibility that they constitute $\widetilde{\mathcal{D}}$-quasi bases. We recall that for $\widetilde{\mathcal{D}}$ a dense subspace of $\mathcal{H}$, we say that the biorthogonal sets $\left\{\psi_{n}\right\},\left\{\tilde{\psi}_{n}\right\}$ constitute $\widetilde{\mathcal{D}}$-quasi basis if, for all $f, g \in \widetilde{\mathcal{D}}$, the following holds

$$
\langle f, g\rangle=\sum_{n \geq 0}\left\langle f, \psi_{n}\right\rangle\left\langle\tilde{\psi}_{n}, g\right\rangle=\sum_{n \geq 0}\left\langle f, \tilde{\psi}_{n}\right\rangle\left\langle\psi_{n}, g\right\rangle .
$$


Inded, let us consider $\mathcal{D}$ defined in (3), which coincides with $\operatorname{span}\left\{\Phi_{\mathrm{n}}\right\}$. Let us moreover assume that

$$
\mathcal{S}:=\operatorname{span}\left\{\Psi_{\mathrm{n}}\right\} \subset \mathcal{D}, \quad \tilde{\mathcal{S}}:=\operatorname{span}\left\{\tilde{\Psi}_{\mathrm{n}}\right\} \subset \mathcal{D} .
$$

We show that $\left\{\Psi_{n}\right\},\left\{\tilde{\Psi}_{n}\right\}$ are $\widetilde{\mathcal{D}}$-quasi basis for $\widetilde{\mathcal{D}}=\mathcal{S} \cap \tilde{\mathcal{S}}$.

In fact, for $f, g$ in $\mathcal{S}$ and $\tilde{\mathcal{S}}$ we have

$$
f=\sum_{n} \psi_{n}\left\langle f, \tilde{\psi}_{n}\right\rangle, \quad g=\sum_{n} \tilde{\psi}_{n}\left\langle f, \psi_{n}\right\rangle
$$

so that

$$
\langle g, f\rangle=\sum_{n}\left\langle g, \psi_{n}\right\rangle \overline{\left\langle f, \tilde{\psi}_{n}\right\rangle}=\sum_{n}\left\langle g, \psi_{n}\right\rangle\left\langle\tilde{\psi}_{n}, f\right\rangle .
$$

Similarly, we may show that

$$
\langle g, f\rangle=\sum_{n}\left\langle g, \tilde{\psi}_{n}\right\rangle\left\langle\psi_{n}, f\right\rangle
$$

Since $\mathcal{S}$ and $\tilde{\mathcal{S}}$ are dense in $\mathcal{D}$ (see [1]), $\mathcal{D}$ is dense in $\mathcal{H}$, and the result follows.

\subsection{Metric operator}

From the procedure in [14] it can be shown that there exists a bounded metric operator for $H$, as a consequence of the reality, simplicity and completeness of its eigenfunctions. Further, $H^{*}$ shares these properties. Nevertheless, any metric operator for this model possesses an inevitable singularity. The analysis of this situation requires the notion of pseudospectrum.

Trefethen and Embree, in their 2005 monograph [21], advocate the use of pseudo-spectra when studying non-self-adjoint operators. The spectrum does not contain sufficient information to derive quantum mechanically relevant conclusions for non-Hermitian operators.

Non-Hermitian operators have spectral instability, with small perturbations changing the spectrum. For instance, complex eigenvalues can appear very far from the original ones. The analysis of pseudospectrum is crucial for correctly understanding the Hamiltonian behaviour. Given a positive number $\epsilon$, the $\epsilon$ pseudospectrum (or simply pseudospectrum) of $H$ is defined as

$$
\sigma_{\epsilon}(H)=\sigma(H) \cup\left\{z \in \mathbb{C}:\left\|(H-z)^{-1}\right\|>\epsilon^{-1}\right\},
$$

where $\sigma(H)$ consists of the complex numbers $z$ for which the resolvent $(H-z)^{-1}$ does not exist as a bounded operator on $\mathcal{H}$. It is known that if $H$ is quasiHermitian, with a positive, bounded and boundedly invertible metric, the pseudospectrum of $H$ is trivial [4]. Therefore, a given operator $H$ with nontrivial pseudospectrum cannot possess a nonsingular bounded or boundedly invertible metric. The Hamiltonian $H$ possesses a nontrivial pseudospectrum [13], so the singularity of the metric operator is unavoidable. 


\section{Conclusions}

Interpreting the imaginary part of $H$ as a perturbation, even infinitesimally small, we conclude that it has severe consequences on the behavior of $H$, as far as eigenvectors of $H$ cease to be a basis of $\mathcal{H}$. In fact, we have proved that the eigenvectors of $H$ and $H^{*}$ originate two biorthogonal complete families of vectors of $\mathcal{H}$ which are not bases.

The eigenvectors of $H$ cannot form a Riesz basis and the imaginary shifted operator cannot be similar to any self-adjoint operator via a bounded and boundedly invertible transformation.

Singular metrics are not adequate to the physical interpretation of non-Hermitian Hamiltonians. Nevertheless, singular metrics may give rise to new physics and mathematics, since the transformed Hamiltonians exhibit new features and open up completely new perspectives.

\section{Acknowledgments}

Thanks are due to the referee by most valuable comments.

This work was partially supported by the Centro de Matemática da Universidade de Coimbra (CMUC), funded by the European Regional Development Fund through the program COMPETE and by the Portuguese Government through the FCT - Fundação para a Ciência e a Tecnologia under the project PEstC/MAT/UI0324/2011.

\section{References}

[1] F. Bagarello, Construction of pseudo-bosons systems, J. Math. Phys. 51 (2010) 023531;

[2] F. Bagarello, Pseudo-bosons, so far, Reports on Mathematical Physics, 68 (2011) 175-210;

[3] F. Bagarello, More mathematics for pseudo-bosons, J. Math. Phys, 54 (2013) 063512.

[4] F. Bagarello, F. Gargano, D. Volpe, D-deformed harmonic oscillators, International Journal of Theoretical Physics 01/2015.

[5] F. Bagarello, J.-P. Gazeau, F.H. Szafraniec, M. Znojil, Eds., Non-Selfadjoint Operators in Quantum Physics: Mathematical Aspects, Wiley, 2015, pp 145152.

[6] N. Bebiano and J. da Providência, Non-Hermitian Hamiltonians with unbounded metric, submitted. 
[7] J. da Providência, N. Bebiano and JP. da Providência, Non Hermitian operators with real spectra in Quantum Mechanics, Brazilian Journal of Physics, 41 (2011) 78-85.

[8] N. Bebiano, J. da Providência , J. P. da Providência, Mathematical Aspects of Quantum Systems with a Pseudo-Hermitian Hamiltonian, Brazilian Journal of Physics, 46 (2016) 152-156.

[9] C.M. Bender and S. Boettcher, Real Spectra in Non-Hermitian Hamiltonians Having PT Symmetry , Phys. Rev. Lett., 80 (1998) 5243-5246.

[10] C.M. Bender, D.C. Brody and H.F. Jones, Complex Extension of Quantum Mechanics, Phys. Rev. Lett, 89 (2002) 27041.

[11] A. Gonzaléz Lopéz and T. Tanaka, Nonlinear pseudo-supersymmetry in the framework of $N$-fold supersymmetry, J. Phys. A: Math. Gen. 39 (2006) 371523 .

[12] T. Tanaka, J. Phys. A. Math. Gen. 39 (2006) L369-L376.

[13] D. Krejčiřík, P. Siegl, M. Tater, J. Viola, Pseudospectra in non-Hermitian quantum mechanics, J. Math. Phys. 56 (2015) 103513.

[14] P. Siegle and D. Krejčiř́ík, On the metric operator for the imaginary cubic oscillator, Phys. Rev. D 86(2012) 12702(6).

[15] F.G. Scholtz, H.B. Geyer and F.J.W. Hahne, Quasi-Hermitian operators in quantum mechanics and the variational principle, Ann. Phys. NY 213 (1992) 74 .

[16] M. Znojil, Should PT Symmetric Quantum Mechanics Be Interpreted as Nonlinear?, J. Nonlin. Math. Phys., 9 (2002) 122-123.

[17] A Mostafazadeh, Pseudo-Hermitian Quantum Mechanics with Unbounded Metric Operators, Cite as: arXiv:1203.6241 [math-ph], Phil. Trans. R. Soc. A (2013);

[18] A Mostafazadeh, Exact PT-symmetry is equivalent to Hermiticity, J. Phys. A: Math. Gen. 36 (2003) 7081;

[19] A Mostafazadeh, Pseudo-Hermicity and generalized PT- and CPT- symmetries, J. Math. Phys. 44 (2003) 974; arXiv:quant-ph/060173v2.

[20] H. Inoue and M. Takakura, Regular biorthogonal pairs and Pseudo-bosonic operators, arXiv:1605.06269 (2016).

[21] L. N. Trefethen and M. Embree, Spectra and Pseudospectra: The Behavior of Nonnormal Matrices and Operators, Princeton University Press, 2005. 